\title{
TOWARDS "DRONE-BORNE" DISASTER MANAGEMENT: FUTURE APPLICATION SCENARIOS
}

\author{
Tullio Joseph Tanzi ${ }^{\mathrm{a}, \mathrm{b}}$, Madhu Chandra ${ }^{\mathrm{c}, \mathrm{d}}$, Jean Isnard ${ }^{\mathrm{b}}$, Daniel Camara ${ }^{\mathrm{e}, \mathrm{b}}$, \\ Olivier Sebastien ${ }^{\mathrm{f}, \mathrm{b}}$ and Fanilo Harivelo ${ }^{\mathrm{f}}$ \\ ${ }^{a}$ Institut Mines-Telecom, Telecom ParisTech, LTCI CNRS. France, tullio.tanzi@telecom-paristech.fr \\ ${ }^{\mathrm{b}}$ URSI France Commission F \\ ${ }^{c}$ Chemnitz University of Technology, Germany madhu.chandra@etit.tu-chemnitz.de \\ ${ }^{\mathrm{d}}$ URSI Germany Commission F \\ ${ }^{\mathrm{e}}$ Criminal Intelligence Service of the French Gendarmerie (STRJD). daniel.camara@gendarmerie.interieur.gouv.fr \\ ${ }^{\mathrm{f}}$ LIM - Univ. de la Réunion, France. \{olivier.sebastien, fanilo.harivelo\}@univ-reunion.fr
}

KEY WORDS: Humanitarian, Autonomy, UAV, Communications

\begin{abstract}
:
Information plays a key role in crisis management and relief efforts for natural disaster scenarios. Given their flight properties, UAVs (Unmanned Aerial Vehicles) provide new and interesting perspectives on the data gathering for disaster management. A new generation of UAVs may help to improve situational awareness and information assessment. Among the advantages UAVs may bring to the disaster management field, we can highlight the gain in terms of time and human resources, as they can free rescue teams from time-consuming data collection tasks and assist research operations with more insightful and precise guidance thanks to advanced sensing capabilities. However, in order to be useful, UAVs need to overcome two main challenges. The first one is to achieve a sufficient autonomy level, both in terms of navigation and interpretation of the data sensed. The second major challenge relates to the reliability of the UAV, with respect to accidental (safety) or malicious (security) risks.

This paper first discusses the potential of UAV in assisting in different humanitarian relief scenarios, as well as possible issues in such situations. Based on recent experiments, we discuss the inherent advantages of autonomous flight operations, both lone flights and formation flights. The question of autonomy is then addressed and a secure embedded architecture and its specific hardware capabilities is sketched out.

We finally present a typical use case based on the new detection and observation abilities that UAVs can bring to rescue teams. Although this approach still has limits that have to be addressed, technically speaking as well as operationally speaking, it seems to be a very promising one to enhance disaster management efforts activities.
\end{abstract}

\section{INTRODUCTION}

\subsection{General introduction}

When a natural disaster occurs in a populated zone, a fast and effective organization of the disaster management is required to assist the affected population, minimize the number of victims and limit the economic impact (Guha-Sapir, 2013). At all phases of disaster management (preparedness, response, recovery and reconstruction) one of the first actions to be taken is to set up a disaster cell for coordination. The detection and the monitoring of the impact of natural disasters on the terrain are mainly performed by space borne and air borne sensors relying on radio and optical instruments (Tanzi, 10) and (Chandra, 14). Contrary to limitations in the time window of observation attached to optical instruments (i.e. no observation at night or in presence of cloud cover), radio observations are available $24 / 7$ and relatively insensitive to atmospheric conditions: these are therefore particularly useful during the "Response phase" of the disaster management cycle when information must be delivered as quickly as possible to the disaster cells (Wilkinson, 10), (Lefeuvre, 13), (Tanzi, 11).

UAVs (Unmanned Aerial Vehicle) may bring significant improvements with respect to these issues. Depending on the requirements of their potential mission, UAVs can easily be equipped with different kinds of sensors, including optical ones. Of course, their altitude permits a higher quality of images and an under-the-clouds observation. Finally, search and rescue teams may carry UAVs and deploy them based on the site needs. For example, flying drones can be used to explore flooded areas in order to find a practicable path to victims. In this respect, UAVs extend the exploration range of rescue teams while at the same time improving their own safety in areas that may be dangerous. A good example is the senseFly UAV (Ackerman, 2013), which during the aftermath of 2010 Haiti earthquake, automatically mapped the region, enabling authorities to quickly draw maps of devastated areas. These maps helped the rescue teams and could improve the lives of victims in the aftermath of the earthquake.

Of course, UAV also have drawbacks and cannot be operated at all time. First of all, they have to cope with weather conditions at a higher degree than conventional aircrafts: because of their light weight and rather low propulsive power, they are more sensitive to wind gusts, for instance. Secondly, they are less prepared to face difficult environments, such as heavy rain (water-proof conception) or hot temperatures. Therefore these basic conditions have to be checked prior to operating them. That said, we make the assumption in this article that they are good enough.

Developing and integrating autonomy features into the UAV is a key to this application. Indeed, the UAV is likely to be in a situation where it will be unable to communicate with the control centre, either sporadically, due to interference, or for an extended amount of time if it explores terrain behind obstacles or beyond the reach of any radio relay. Depending on the realtime requirements, communication capabilities, and complexity of the deployed sensors, the collected data could be partially processed on board. Appropriate navigation or data fusion 
algorithms have to be developed. Autonomy does not mean that the UAV will not receive instructions from outside, for instance in order to zoom in on some scenes that could be of interest of the control centre, even if the UAV was not considering to do so in the autonomous mode.

Autonomy plays a key role in this application, but specific features have to be developed in order to look for victims. An appropriate range of detectors will have to be combined in order to distinguish between human beings and inanimate objects, especially when victims are buried under debris and cannot be detected optically. The UAV should also be able to discriminate victims from rescue teams. Algorithms have to be adapted to the detection and monitoring of victims and groups of victims in order to anticipate their movements as well as considering if they require medical treatment.

As explained in (Tanzi, 2009), new approaches and the use of new technologies are required for a more efficient risk management, before, during, and after a potential crisis. Every specific action at each step of the crisis must be taken into account. For that purpose, new dedicated tools and methodologies are required to enhance the handling of crisis situations.

\subsection{Related works}

The numbers of use cases where drones have already been useful in humanitarian settings are numerous; the ones described hereafter are just a small subset of these for illustration purposes. Danoffice IT, has a commercial drone solution for disaster response (Pedersen, 2014). It was used in real operation sites such as the typhoon Yolanda in Tacloban, Philippines, where it helped in the identification of the operation site, and on the identification of feasible roads. In the same disaster, the CorePhil DSI team (Meier, 2014) used a fixed wing drone, eBee, to capture aerial imagery of downtown Tacloban. These images were further analysed through crowdsourcing and helped in the generation of the most detailed and up-to-date maps of the region. These maps were afterwards used by different humanitarian organizations and even by the Filipino Government.

The control of fleets of drones is also not a new theme. In fact, it is a well-studied subject in the military context. Of course the purpose is here different; the same goes for the flight control. However, even on military operations the proposed fleet control mechanisms intend, basically, to help humans to control the drones rather than providing a fully autonomous fleet. For example, (Cummings, 2007) proposed an automation architecture to help humans on the supervision of a drone fleet, but the drones are not completely autonomous, it is still up to the human operator to decide the drones mission. The same comments are valid for other works in the field, e.g. the work of Arslan and Inalhan (Arslan, 2009), where the whole effort relies on helping one operator to control multiple drones.

\subsection{Examples of Drone applications}

In a disaster scenario, drones can perform a number of different tasks to help in the relief effort. Tasks may vary from providing means of communication to the creation of high-resolution maps of the area and the autonomous search for victims. Maintaining communication over disaster areas is challenging. One cannot just rely over the public communication networks: first because these may be unavailable in remote areas and, second, because even if they are available the network may be damaged or destroyed. Nevertheless, the coordination of the relief efforts requires communication. Drones can work as temporary mobile access points for extending the coverage in affected areas. This service may be offered not only for the rescuers, but also for the general population with the creation of small picocells. For example, after hurricane Katrina, at New Orleans, the public network was out of service and Verizon, the local provider, granted to the first responders the right to use their frequencies.

Another important task that can be autonomously performed is the creation of high-resolution maps of the affected area. Disasters may change drastically the affected region, which may void previous maps completely. Drones can fly over the region with 3D cameras and, with the help of GPSs (Global Positioning Systems) and publicly available relief maps of the region, automatically create up-to-date $3 \mathrm{D}$ maps of the area. These maps can be used to understand the impact of the disaster over the region and, for example, decide which roads need to be closed, which are the best paths to reach the most damaged areas or even help in the planning of the relief supplies delivery. Among active roles they could play to help in the search and rescue operations, we can highlight the infrared scan of the region, the use of ground penetration radars or the systematic search of mobile phones by the detection of their specific radiation.

\subsection{Regulation and legal aspects}

Although the research work presented in this article mainly focuses on technological and scientific aspects, it is necessary to take into account the regulatory framework that controls the use of UAVs. Actually, this legal framework is quite different from a country to another. Globally speaking, it is rapidly evolving and tries to find a compromise between a highly restrictive approach that prohibits most UAV operations that are not declared by a certified pilot and a freer one.

For instance, in the United States, regulation evolved in late 2015 to make UAV registration mandatory along with a declaration of ownership (Federal Aviation Administration, 2015). Also, manufacturers must now include no-fly areas in embedded software to avoid sensitive secured area fly-by and pilots are requested to keep their drone in the line-of-sight.

All these rules could be severe barriers against the concept of autonomous aerial vehicle we would like to present here. However, one must take into account the fact that the conditions that apply in normal circumstances are not the same when a disaster strikes. Under these particular circumstances, special authorizations can be obtained in a particular framework, which is controlled by experts from all sides: rescue teams, governments, communication providers, local authorities, etc. Malone et al. (Malone, 2006) describes a good example of such a specific process that has been set to address a crisis, in this example, hurricane Katrina, as evoked before. At that time, many specific exceptions where made in multiple domains and more particularly frequencies allocations, communication routing and flight regulation. There is no doubt that this framework can be updated to allow the use of autonomous UAVs, but this has to be integrated at the early stages of the project.

\section{UAV RELIEF REQUIREMENTS}

One of the first actions to be taken is to set up a disaster cell for coordination. For major risks, this includes links with national 
ministries, civil defence, regional and local administrations, non-governmental administrations involved in disaster management as well as experts, crisis staffs, a command chain, an information chain, etc. During such an event, maintaining a communication link with rescue workers on one hand and with victims on the other hand is crucial. Unfortunately, whenever the public communication infrastructure is damaged, rescue teams rely essentially on radios or satellite communications.

UAVs might extend the communication range available as they may be deployed as mobile radio relays. UAVs may also convey messages working in a Disruption Tolerant Network (DTN) way. Of course, the operation of a UAV generates its own communication needs, and an UAV control centre must be put in place either by the disaster cell or by mobile units on site. The operation of such a centre should be as seamless and adaptable as possible. This can only be possible by making the UAV navigation autonomous.

Data sensing results have to be communicated as they are produced, and will serve for the coordination of relief operations. In this sense, the UAV should also be autonomous in deciding which data to pre-process and to communicate in order to establish operational priorities. Communications between a control centre and UAVs and between UAVs must be secured to prevent any unauthorized access to the sensitive data that flows through the network.

\section{Data integrity preservation concerns}

Accessing the data collected by the UAV (Wilkinson, 2010), (Lefeuvre, 2013), (Tanzi 2011) must be managed by the control centre. Data should be preserved and secured during operations as well as in case of a crash. Actually, data sensed may be valuable commercially or may have political implications. The deployment of UAVs should not be diverted by third parties and ultimately result in hampering the relief operations.

\section{Search operations}

Similar to terrain reconnaissance, satellites and aircrafts are currently used to evaluate the consequences of natural disasters, with equally problematic liabilities in terms of weather and diurnal conditions, as well as availability. Low altitude teledetection (drone-based or rover-based) enables a sharper approach, which allows more precise victim localization and counting. Safety issues are a major concern in this application. The low altitude and autonomy of navigation of a UAV may potentially cause injuries to nearby victims or rescuers in case of a crash, for instance. This means that UAVs must encompass this dimension from the very early stages of their design and integrate safety mechanisms in order to handle possible mechanical, hardware and software failures. It is, for instance, possible to operate the UAV in a degraded mode reduced power in order to safely land the drone or to fire a parachute to reduce the impact of the descent.

The security of the data sensed and stored on-board UAVs may be especially sensitive with respect to victims' privacy. For instance, there have been situations in the past where pictures of recognizable victims have made the headlines without their agreement.

The deployment of UAVs for such applications will also bring up societal challenges. Indeed, the appearance of an UAV may be terrifying to an unprepared victim, which might reduce the effectiveness of the detection operations. In contrast, victims may not notice UAVs flying at a high altitude and therefore they may fail to signal their position, as they would have tried to do for an aircraft. New standards will probably have to be defined in this respect.

\subsection{Payload specifications}

Previous works with specialists from the disasters intervention (French "Protection Civile", Médecins sans frontière (MSF), International Committee of the Red Cross (ICRC), etc.) allowed us to formalize three main requirements for rescue teams, among the set of applications described in the previous section. The main goal of the rescue teams is to assist victims in the shortest time frame possible. To fulfil objectives, it is necessary first to detect the people impacted by the event, and second, to identify the possible accesses (e.g., safe roads and practicable paths) to the disaster area and to the victims. These operations are not instantaneous, and, third, it is necessary to perform a continuous assessment of the evolution of the situation in the impacted area. In this phase, we need to inform and reassure victims so that they keep calm and avoid getting into useless dangerous situations. The effectiveness of these operations depends on the speed and accuracy at which they can be carried out.

We will now discuss the payloads issues and the associated data processing capabilities that can be put on-board UAVs to illustrate the interest of this technology for addressing the previously mentioned challenges.

\subsection{Systematic terrain scan}

A first class of UAV data sensing payloads, is related to the systematic coverage of an area in order to perform a "rapid mapping" of the target zone. Emergency situations typically requires an appropriate over time monitoring, and at some points of interest, to rely on a more detailed analysis using veryhigh resolution data. Using such information, it is easy to produce in few passes a thematic map appropriated to the relief operations. The interest of these maps for decision support is very dependent on the sensor used. For example, the "LIght Detection And Ranging" (LIDAR) is a technology that uses laser pulses to generate large amounts of data about the physical layout of terrain and landscape features. All varieties of LIDAR operate using the same basic principle. The instrument fires rapid pulses of light (laser pulses) at the landscape and a sensor mounted on the instrument measures the amount of time taken for each light pulse to bounce back. Because light moves at a constant and known speed, the instrument can then calculate the distance between itself and the target with high accuracy. By rapidly repeating the process, the instrument is capable to build a complex "picture" of the target terrain. With this method, we can obtain a refined Digital Surface Model (DSM) allowing a large set of ground analysis (see Figure 1).

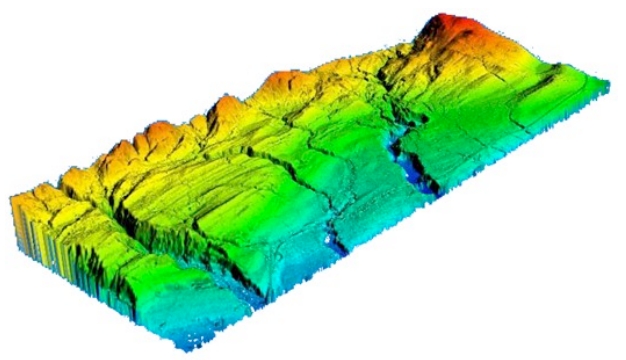

Figure 1. Terrain mapping with a LIDAR

We chose to focus our efforts on a LIDAR-based environment reconstruction over a dense-DSM acquisition based on a photogrammetric approach because it produces $3 \mathrm{D}$ meshes, 
which are easier to process on an embedded computer (in comparison to raster images) and because this approach can be used both under poor lighting conditions and indoor (inside a building or in a basement).

The terrain coverage task is a new feature supported in a very flexible way by the use of UAVs. This basic building block also offers a strong interest to deploy more complex payloads and processing capabilities as explained in the next two sections.

\subsection{Autonomous detection and classification}

A second class of data sensing payloads aims to detect and classify of victims, see Figure 2. From the drone side, it means advanced people detection capabilities. Simple people detection (e.g., silhouette based) is not sufficient. Indeed, the quantification is important information for organizations that manage the disaster. It allows the optimization of logistical aspects (tents, food, medical staff, etc.). It is therefore necessary, upon the detection, to implement a phase of recognition (signature) in order to count each victim only once. Another related problem is the discrimination between victims and members of the relief team (see Figure 2).

An UAV will have to identify groups of disabled persons, and determine, for example, whether they are adults or children. To perform this, we are currently working on a simple principle based on Closed-Circuit TV (CCTV) techniques: using image recognition algorithm, the system can detect a body shape and draw a bounding box (see Figure 3) (Apvrille, 2014), (Tanzi, 2014). By analysing the size and ratio of the box, we try to characterize a person. This distinction makes sense because the support that rescue teams have to provide strongly differs in the two cases. Such sorting triage must be compliant with international and local ethical policies. Furthermore, tracking a specific group might help in determining their speed and expected position in a near future.

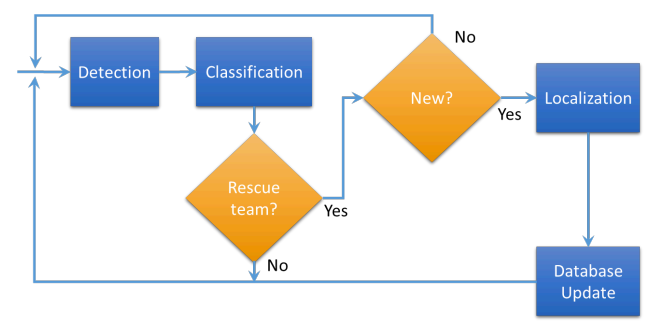

Figure 2. Detection cycle
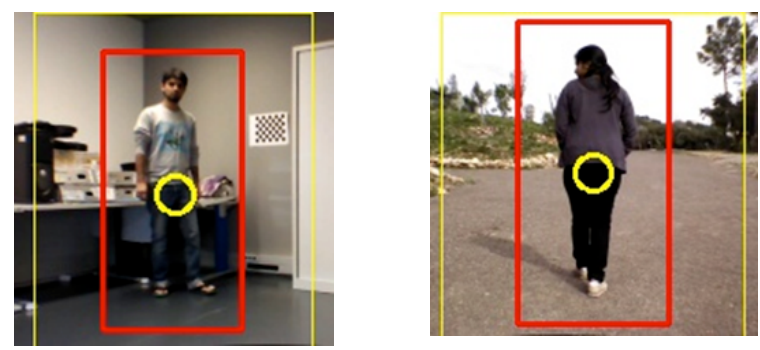

Figure 3. Bounding Box principle

\section{Data fusion}

Data fusion between optical (visible and InfraRed (IR)) and Radar data can also produce non-conventional data allowing the extraction of pertinent information for decision support.
This is now possible thanks to the increase in the processing power of processors, at a lower energy consumption of active components (including radio frequency) and the evolution of the memory chips, which have larger capacities into smaller volumes. This is a highly significant factor for the on-board equipment. Embedded sensors will have multi-band capabilities, so as to consider all radiation sources. They will also feature a strong directivity in order to precisely target the source of an emission. Finally, they have to be low weight. Sensors will be used for UAV navigation as well as for terrain mapping and victim detection.

Another non-conventional approach to detect buried victims is to search for the electromagnetic emissions of their mobile devices. The objective is to identify the points under the rubble where the rescue teams are most likely to find victims. It highlights the need for a new airborne solution to detect and map the position of people. The main idea is to make an image of the ground using an antenna, carried by a drone flying at a very low altitude. A typical scenario based on this approach will be presented later in this article.

\section{DRONE ARCHITECTURE}

Independent of the mapping, sensing or scanning methods one wants to apply, autonomous drones need to communicate to organize themselves. All drones, regardless of their type, should be able to communicate with others and autonomously coordinate the actions to divide the tasks to be done. The layered organization proposed in the Figure 4 represents this common internal organization. Even though the implementation may change to consider the specificities of the drone, each one of the activities represented at Figure 4 needs to be implemented by all the drones.

The role of each one of these boxes is as follows: the MAC (Medium Access Control) layer provides the network abstraction to all the other modules. It contains the specifics of the network technology used and can be interchanged to adapt to local regulations and standards. The radio management subsystem is responsible for controlling the power of the radio and optimizes the communication with the other drones. The selforganizing network module is responsible for exchange messages with the nearby drones to coordinate the efforts and divide tasks. The information relay task is responsible for receiving data from the other drones and either forwards it to the next drone in the direction of the destination, or delivers it to this final destination.

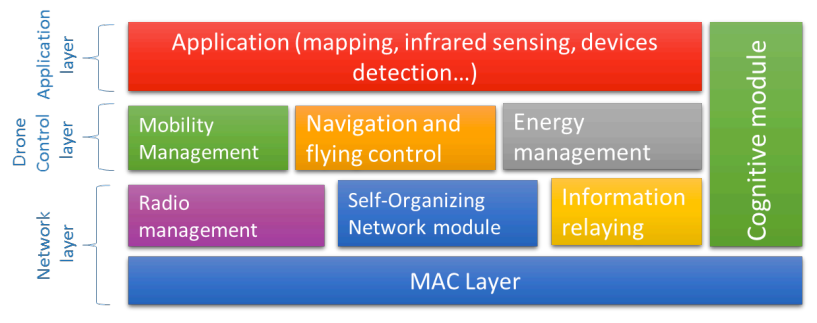

Figure 4. Layered organization of modules common to all drones, independently of their type (Câmara, 2014).

The mobility management module is responsible for planning the mobility of the drone considering the objectives and the probable actions of the other drones in the region. The navigation and flying control module is responsible for implementing the planning done by the mobility management module. Based on geographic information, e.g. Global 
Positioning system (GPS) data, it controls the route and the power of the engines. This architectural component guarantees that the drone will fly in the right direction and speed. The energy management module is in charge of keeping track of the remaining energy and issuing warnings when it is time for the drone to return to the base. In case the energy gets critically low, this module is also responsible for starting the emergency procedure. The emergency procedure, among others actions, consists of sending a distress message with the current position and safely landing the drone while repeating the distress message at regular intervals.

The application layer is dependent on the task to be done at the time, the kind of drone and the type of sensors available over the drone. The application should also be interchangeable since the tasks for the drones may evolve during the rescue operation effort. The cognitive module, vertical to all the others, provides generic Artificial Intelligence (AI) algorithms that help on the decision-making activities of all other modules. For example, the mobility management module can use it to try to infer the actions other drones will take to optimize the coverage of the area. The energy management module can use it to decide the best moment to return to the base. i.e. based on the energy consumption, how much energy the drone should spend to fly from the point it is to the base.

\subsection{Fleet Architecture}

Considering the work they are capable of performing, even one drone is already able to provide great help to the relief effort. However, to be effective and use the drones' full potential, more than one kind of drone is required. This section proposes an autonomous communication and coordination architecture to enable the efficient use of different kinds of drones, and their specific characteristics, over disaster scenarios. The main architecture is depicted in Figure 5.

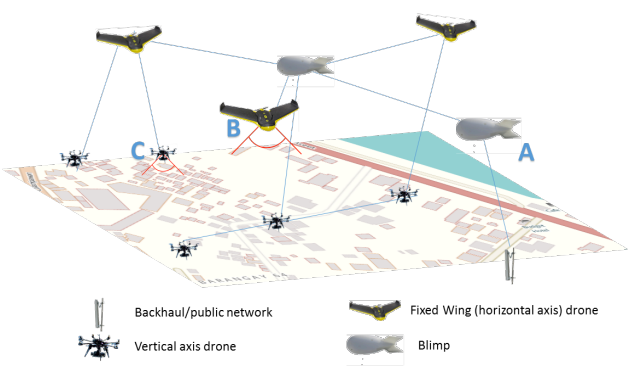

Figure 5. Multi-layer organization of the drones' fleet, highlighting the instantaneous communication links.

Blimps (A) have a higher autonomy and stability. Fixed wing drones (B) present also good energy efficiency and can fly to a relatively high altitude, which makes their vision angle larger than the ones of vertical axis drones (C). Vertical axis drones, even if they present lower energetic efficiency, have a better manoeuvrability and can adapt their speed to the characteristics of the terrain.

The proposed drones organization is composed of three different specialized drones performing different tasks over the first hours, just after the crisis outbreak. The three categories of drones are blimps, fixed wings (horizontal axis) and rotary blade (vertical axis) drones. We consider that drones are constantly aware of their autonomy and the required energy to reach the closest recharging station. When one of them perceives that the amount of remaining energy is getting close to the limit, it warns the nearby drones that it needs to leave the formation. This warning also implies that, depending on the activity it is performing, another drone will need to take over its duties. Nearby drones will then organize themselves, and if required, decide which one will take over the interrupted task. This is a basic principle and applicable for all three types of drones.

One of the main tasks drones may perform is to provide temporary communication infrastructure. Stable and low energy devices should compose the main backbone infrastructure. Ideally, blimps could perform this role quite well. They are stable and, given their characteristics, possess a much larger autonomy than the average drone. For example, the X-Tower from Stratxx can fly for 23 days broadcasting 12 channels of digital TV during this entire time (Stratxx, 2014). Flying at a relatively high altitude, blimps have the advantage to provide a large footprint coverage. The covered area can be further extended by interconnecting multiple blimps. To avoid interference, backbone nodes should have two interfaces, one to work as access point to other nodes, and a second interface dedicated to handle the backbone traffic, i.e. routing other backbone nodes traffic and accessing the backhaul. Any other node of the region, user's equipment and even other drones, could use the deployed infrastructure to exchange data.

Fixed wing drones have a lower autonomy than blimps, but they have the advantage of being able to cover the region faster. Equipped with GPS, 3D cameras and trusting open geographical databases, these drones can provide the data for the automatic generation of an up-to-date map of a relatively large region in a matter of minutes. Such maps can be further distributed and used by different agencies on the coordination of the research and relief efforts. The fixed wing drones can coordinate themselves directly, through the blimp backbone, or even through the ground backhaul, if they are in its communication range. This coordination is important to grant a full and optimal coverage of the region by the fixed wings drones. Given its characteristics, the most rational way to divide the areas are in strips, so that the drones can go over the strips one by one doing only one turn at the end, as depicted in Figure 6. The advantage of dividing the area is also to be able to divide the work among different drones, if available (Câmara, 2014).

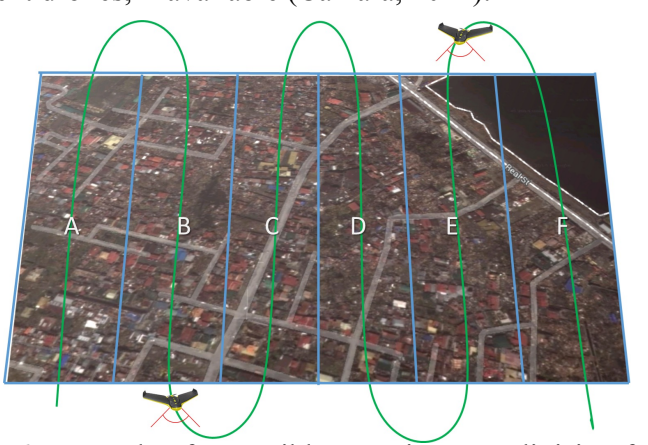

Figure 6. Example of a possible mapping area division for fixed wing drones.

Vertical axis drones have, typically, a smaller autonomy than blimps and fixed wings drones. However, they have other advantages: first, they are able to fly in every direction, horizontally and vertically, as well as hover in a fixed position. Moreover, they can also fly steadily close to the ground, to provide high fidelity data from Ground Penetrating Radars (GPR) and sense weak portable devices signals. Given its characteristics and limited autonomy, to be effective, horizontal blade drones should concentrate their efforts over the spots of 
interest, e.g. collapsed buildings, avalanche and mudslides runout zones. Those spots of interest for these drones can be either provided by the rescue teams or automatically detected from the high fidelity maps generated by the fixed wing drones. It is also important for these drones to coordinate to provide a full, coverage of the target area with non-overlapping, for 2D maps. If the objective is a $3 \mathrm{D}$ reconstruction, the reconstitution should be done with the least overlap as possible between different drones, as the photogrammetry requires an overlap between images. As depicted in Figure 7, taking into account the high fidelity maps, we can automatically generate a grid attributing an identification to each zone: e.g. D2, after covering a zone can notify others that it has been scanned successfully and reserve another non covered area nearby. The advantage of this technique is that areas with low or no interest, e.g. G1 on Figure 7, can be scanned much faster than other areas, e.g. C2, which have much higher density of interest spots. These dense areas can even be further divided, since what matters is to divide fairly the work to be done, not the area itself. It is expected that some areas will be covered faster than others. Drones that decide to subdivide their zones into a smaller granularity grid, inform other drones that they have more areas to work on, e.g. C2.A3. To choose one area, drones can base their decision on a number of factors that may include: distance from the present position, flight plan, amount of interesting spots, available energy level, and other drones probable route. If a drone is forced to leave an area before being able to fully cover it, e.g. low energy, it divides the area and warns other drones about the sub-areas it already covered. The remaining areas go to the "work to be done" pool and will be later cover by other drones. Ideally the negotiation over which areas were already covered and which are the next should be done autonomously and directly between the drones (Câmara, 2014).

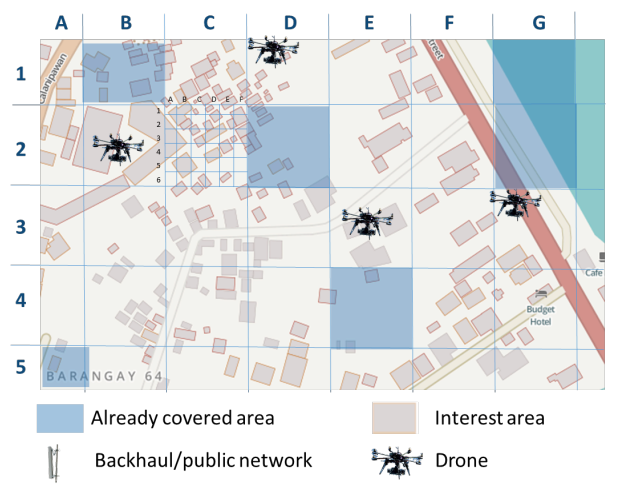

Figure 7. Drones objectives and sub-objectives division

The data acquired by the drones need to be constantly sent to the operations centre via backhaul (an intermediate link between backbone network and smaller subnetworks) or public network. It is possible to filter part of the control data to save bandwidth and energy. Only the important pieces of information, required for decision making, is sent e.g. covered areas, drones energy level and forecasted autonomy. Other data, such as selforganizing controlling messages can be confined to the drones' network.

Drones should be as autonomous as possible, but also should be able to receive instructions remotely, for example, to provide information of specific areas that has special interest to the teams. In some cases, the operations centre may require, for instance, an online video transmission of a specific area. If the drone is far away, the video stream is sent, in a multi-hop way, to the operations centre. In case the drone loses its connection during the transmission, the data is relayed and sent to the operations centre at either the next time the drone is connected or, in an opportunistic way, to a nearby drone that is perceived to be going to the direction of the backbone or the backhaul. One cannot trust that network will be always fully connected: store and forward mechanisms (Polat, 2011) need to be implemented and should be part of the basic communication mechanisms.

As of early 2016, the autonomous flight controller depicted in figure 4 has been implemented and is able to pilot a drone that autonomously looks for a person and tracks him/her. We are currently working on the next steps to add other actions evoked here: fleet management, real-time mission re-assignment and intelligent communication relaying. This leads us to work on both the hardware and software sides in order to embed enough processing power, required sensors and interfaces, which are devices that tend to drain battery more. Therefore, it is necessary to run only a subset of available components to reduce power consumption.

However, there is no doubt that technological limitations regarding these needs will be overcome, so we can already discuss of a practical terrain scenario.

\section{TYPICAL USE CASE: ELECTRO MAGNETIC (EM) WAVES DETECTION}

In this part, we will illustrate our approach by presenting a wave detection process dedicated to the localization of buried people after a disaster such as an earthquake or a tsunami strikes. This process is a work-in-progress: its implementation requires that the development work described in the previous part is completed in order to be tested in a real situation.

The following initial hypothesis is made: nowadays, people tend to own one or more wireless personal devices (smartphone, smart watch, bluetooth headset, etc). Therefore, if one of their particular Electro Magnetic (EM) signatures is detected, chances are high that their owner is located at a very close distance away.

\subsection{Overview}

The idea is thus to have a devastated area automatically browsed by drones fitted with specific antennas to stimulate the circuits embedded by those devices and measure a response to identify places where victims are and draw a map of priority operations (for instance area where density of detected devices are higher). Figure 8 shows an overview of the process for one buried victim.

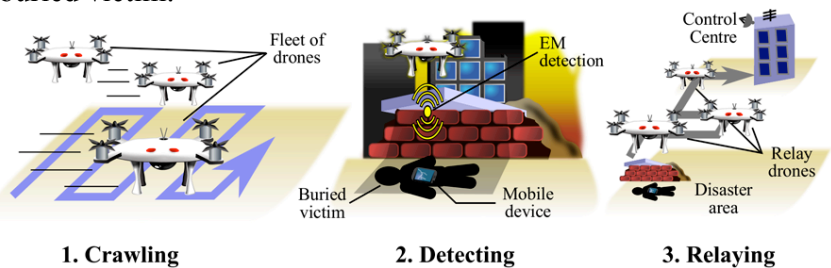

Figure 8. Automated victim localisation process

During the crawling operation (step 1 of the figure), a fleet of drones explore the devastated area according to the pattern defined in Figure 6. Each of them is fitted with various antennas able to scan for the main consumer wireless EM bands that will be detailed in a forthcoming part. 
Once a response is obtained (step 2 of the figure), an attempt to communicate using a higher layer of the OSI model (Brisco, 2000 ) is realised. This would allow gathering more information from the device by actively communicating with it (Sébastien, 2015) enabling a request/reply dialogue, with or without an action from the owner. Users more and more tolerate this particular point. Thus, during Paris terrorist strike in November 2015, Facebook deployed its Safety Check service allowing people to attest very quickly to their relatives that they were safe. However, this is not a necessity for EM detection as passive scanning may be enough to fill a report. Finally, when drones have finished performing their tasks, they can reorganise themselves to act as routers in order to transfer all the data to a control centre (step 3 of the Figure). Time is indeed precious, so it might be a problem to wait for the whole fleet to fly back to the station. In-air relaying allows sharing the data from one drone to another until the operations centre is reached.

One of the keys in EM waves detection is the capacity to look for low-energy signals. Indeed, on one hand rubble can dramatically attenuate them, and on the other hand, chances are high that victims devices cannot run at maximum emitting power, either because they are damaged (the case embedding the antenna is broken) or running short of battery and thus operating in energy-saving mode. This can be solved in two ways. Firstly, drones can operate at a lower altitude than most human-operated aircrafts. Secondly, we rely on hi-gain passive antennas from the drone side. To improve source location, we expect to use synthetic aperture techniques: the basic idea is to rely on irradiating or collecting devices spread in a rather large area (for an antenna), whose signals are combined coherently to obtain a resolution as high as a corresponding large antenna. Drone movements can also be used to increase the dimension of the virtual antenna, which is an approach similar to the one used in Synthetic Aperture Radar.

In order to implement this process, it is necessary to have a deeper look at, on one hand, the features of the devices that have to be detected and then, on the other hand, to review the networking interfaces they rely on.

\subsection{Connected personal devices typology}

As we made the assumption that most mobile device can be associated to one person at a time, we need to present those devices in order to set the relationship and hierarchy between them. Thus, sensing one of them from an EM perspective can reveal the presence of others.

The most common device is the mobile phone, and more particularly, nowadays, smartphones as their sales has dramatically increased those years according to Gartner (Gartner, 2015). They are fitted with multiple wireless networking devices, which rely on compatible antennas to support protocols that will be evoked thereafter. Smartphones are also considered as the central point of the personal network because they can handle local (i.e. bluetooth and Wifi) as well as distant communication $(3 \mathrm{G} / 4 \mathrm{G})$. Other radio-devices therefore rely on them to transfer data to Internet for instance. Another common device is the headset, an equipment which allows the use of a mobile phone without holding it. It uses bluetooth connectivity to exchange mostly audio data with a mobile phone. Such a device must not be far from the gateway phone to work correctly. The same is generally true for more recent (and therefore quite rare for the moment) devices: smart watch and connected bracelets. They operate the same way as the headset, using a local personal networking protocol to reach a "smarter" device processing data and possibly relaying it to distant servers.

From an EM point of view, this centralisation can be used to confirm or invalidate the presence of a body: if the signature of a bluetooth headset antenna is detected next to the signatures of the typical antennas of a smartphone, then chances are high that somebody is there, possibly buried under rubble.

\subsection{General Public Networks}

Nowadays user devices are commonly equipped with one or more of the following communication technologies:

Global System for Mobile Communications (GSM, second generation), Universal Mobile Telecommunications System (UMTS, third generation) and Long-Term Evolution (LTE, fourth generation) are telecommunications standards used by mobile phones. They provide the maximum range (of the order of $\mathrm{km}$ ) with an average speed (LTE proposes a downlink peak of $300 \mathrm{Mbits} / \mathrm{s})$. Telecommunications standards rely on an infrastructure run by operators.

Wi-Fi (IEEE $802.11 \mathrm{a} / \mathrm{b} / \mathrm{g} / \mathrm{n} / \mathrm{ac}$ ) is adapted to a domestic use, especially for a high-speed network access (of the order of gigabits/s for IEEE 802.11ac depending on the configuration). It supports an average range (approximatively $40 \mathrm{~m}$ for interior with IEEE $802.11 \mathrm{~g}$ ). The device goes through an access point to communication with the rest of the network.

Bluetooth addresses personal-range communication. It was initially designed as a replacement for cable. It provides a low range (up to approximatively $10 \mathrm{~m}$ for Bluetooth 2.0 EDR class 2) and speed (up to $25 \mathrm{Mbits} / \mathrm{s}$ for Bluetooth 4.0 ) but consumes less energy than the two other standards. Bluetooth permits device-to-device communications.

While Bluetooth presents an interesting option because of low energy consumption, its short transmission range and weak radiated power limit its use in the detection of buried people. EM waves generated by a buried Bluetooth device are likely highly attenuated by rubble. However, coupled with a Wi-Fi or a telecommunication technologies-capable device, a Bluetooth device (e.g., activity tracker or bracelet) can provide precious and accurate information about the victims.

To tackle the potential presence of obstacles along with the crawl and scan strategy, the communication technology must exhibit a high penetration capability despite signal attenuation and interferences. WiFi, GSM, UMTS and LTE perform efficiently in similar situations. I-LOV system (Hamp, 2014) proposes the detection of inactive cellular phones. Such devices are equipped with radio frequency resonators connected to the antenna, which can store energy. This energy can transport signal when the resonators are sent an excitation. Those components echo back the triggering signal. In the experiments, the authors generate a one microsecond burst signal at the frequencies of the GSM900 cellular phone band. It is fed to a device in a testing chamber. The reflected signal is then captured and analysed. Four types of cellular phones were tested. Resonance was observed around $920 \mathrm{MHz}$ and around $950 \mathrm{MHz}$ frequency bands. The authors of (Zorn, 2011) describe a system for the localisation of active GSM phones. A jammer generated by a Software-Defined Radio Base Transceiver Station (BTS) is also used to disable normal communications. Cellular phones are thus forced to register themselves with the BTS. The observation of such registrations indicates the presence of user devices and potentially, their owners in the vicinity. Another study was conducted to check 
the feasibility of locating lost persons, e.g., hikers, from the air (UAVs) by detecting the WiFi signals from their mobile phones. The Wi-Fi adapter of those devices periodically scans for Access Points. A first option is a passive detection of corresponding generated Probe Request frames. A second alternative consists in actively sending Request To Send (RTS) to the target phone. The device if active will respond with Clear To Send (CTS) frames. However, this approach requires the prior knowledge of the physical (MAC) address of the device. Experiments demonstrate the feasibility of the detection of WiFi-enabled mobile phones from the air at distances up to 200 m. Device-free Passive (DfP) (Wang, 2013) and (Moussa, 2009) localisation proposes the positioning, the detection and the tracking of individuals or objects using WiFi. The propositions exploit indoor WiFi network. A radio map is constructed initially. Then, subsequent changes in the environment are detected by monitoring and processing received signals. Indeed, people and entities disturb the pattern of radio waves and consequently introduce changes in the map.

\subsection{Scenarios}

Two approaches of EM detection can be adopted. The "static" approach will consist of designing an antenna whose directivity, gain adjustment weight performance will be optimal according to the available weight and space on-board the UAV. The drone will thus be able to detect an EM emission when being in vertical position. It can then record and transmit the location of the source of EM emission using another dedicated communication to the control centre. In contrast, the "active" approach, will allow, in a single pass, to cover a wider geographical area. To do so, active electronics will achieve a space electronic scanning of an angular area around the vertical to the aircraft, in the plane perpendicular to its displacement. In the case of detection, the UAV will then forward its current coordinates, as well as the angle of signal arrival, to the operations centre. We use the idea of synthetic aperture antennas but in a purely passive approach.

\section{CONCLUSION}

The search and rescue surely enough can make usage of drones for a fair amount of activities. However, if not autonomous and capable of self-organization, these elements can be more a burden than helpful in a catastrophe scenario. Rescuers must focus on the activity they have at hand: that is, saving lives. It is not their job to spend their time handling drones and their tasks. The proposed architecture intends to provide the organization required for a fleet of drones to autonomously, at the push of a button, scan the region and provide useful information. Another intention of the proposed architecture is to use this fleet to provide communication over disaster areas, even for severely affected areas.

It is also important to notice that drones should be able to perform opportunistic communication and coordinate with the nearby nodes. In a disaster scenario, store-carry-and-forward techniques may be the only way to convey important information among the computational elements. Drones can exchange information with each other about the route, and strategies they are taking. If they are, for example, moving in the direction of the operations centre, they can carry the messages of other drones until their final delivery at the destination (Lefeuvre, 2013).

Different kinds of drones may provide different services and, ideally, should play the roles they fit the best. Even though we could exchange some of the tasks among the different drones, it would have an impact on the end results. For example, we could without a doubt use fixed wing drones to create a mobile backbone. However, not only would the organization of the drones to provide constant full coverage be more complex, but also the lifetime of the backbone would suffer.

Among all the high tech objects of our modern environment, drones have an impressively high potential to offer fast and efficient responses in rescue conditions, even if some difficulties must be tackled. The new applications, such as the intervention in hostile environments, require an effective autonomy of mini drones concerning the energy (duration of the mission) and the control-command (decisional autonomy). Hardware and software issues have to be addressed: which algorithmic architectures to adopt? Which embedded system configuration is the most suitable one? Which kinds of Interface are the most appropriate for victims, being in front of the drone? How can a drone help people in critical conditions or provide useful information?

The design of a civilian UAV intended for intervention in postdisaster conditions is an important challenge. The gain in autonomy of drones, coupled with the use of non-conventional sensors such as Lidar, IR camera, etc. will strongly increase response capabilities of the rescue teams on the ground, e.g. people detection, rapid mapping, damage estimation. To be effective, these customized sensor systems must perform their duties in an independent manner and be able to communicate their data to the command centre. This information will then be inserted into the decision making cycle. It is also imperative that the manipulation of these systems does not require any special skills. This condition is an indispensable one, which explains the rationale of our focus on autonomous flight and mission. Without that capacity, it would be not possible to correctly integrate these new tools within the activities flow of rescue teams.

The next step consists in leading field experimentations to acquire data to validate the approach. Indeed, so far we were not able to anticipate certain behaviours. For instance, a typical mobile phone is supposed to look for a network while none is detected. This search requires the device to use full power, which drains a great amount of battery energy. Therefore, some manufacturers limit the time window of this full power mode. We thus have to check if this kind of policy is still compliant with the aim we try to reach. Another aspect concerns the adaptation of the approach considering the geographical area. Disasters can strike everywhere, so the question is to determine how such a system can behave, for example, in developing countries with emerging network infrastructure, or its compliance with the different local communication standards. Much remains to be done to reach an optimal operational use of drones. Usage of sensors has to be adapted to current conditions. As far as instructions for use are concerned, international agreements are requested: i) several national administrations are opposing the identification of a dedicated harmonised frequency spectrum for Public Protection and Disaster Relief (PPDR) favouring a flexible solution which would enable national agencies to choose the most appropriate solution to meet national needs (see ITU-R Resolution 646 (Rev.WRC-15) \& Resolution 647 (Rev.WRC-15)), ii) agreement from ICAO is indispensable for using drones in any non-segregated space (see ITU-R Resolution COM4/5 (WRC$15))$. 


\section{REFERENCES}

Ackerman, E., 2013. "Drone Adventures Uses UAVs to Help Make the World a Better Place," IEEE Spectrum.

Apvrille, L., Tanzi, T. J., Dugelay, J.-L., 2014. "Autonomous Drones for Assisting Rescue Services within the context of Natural Disasters," in XXXI General Assembly of the International Union of Radio Science, Beijing China, p. 4.

Arslan, O., Inalhan, G., 2009. "Design of a Decision Support Architecture for Human Operators in UAV Fleet C2 Applications", 14th, International Command and Control Research and Technology Symposium (ICCRTS), Washington, DC.

Brisco, N., 2000. "Understanding the OSI 7-layer model”, PC Network Advisor, vol. 120, no 2.

Câmara, D., 2014. Cavalry to the Rescue: Drones Fleet to Help Rescuers Operations over Disasters Scenarios, IEEE. Conference on Antenna Measurements \& Applications (CAMA), Antibes, Antibes Juan-les-Pins, France, November $16-19,2014$

Chandra, M., 2014. “'Overview of Modern Multi-parameter Methods of Radar Remote Sensing in Context of Disaster Management" in XXXI General Assembly and Scientific Symposium of the International Union of Radio Science (URSI GASS 2014). ISPRS-URSI Disaster management special session. August 17-23, Beijing, China (CIE).

Cummings, M.L., Bruni, S., Mercier, S., Mitchell, P.J., 2007. "Automation Architecture for Single Operator, Multiple UAV Command and Control", The International C2 Journal, Vol 1, No 2, 1-24.

Federal Aviation Administration (FAA), 2015. Unmanned Aircraft Systems, http://www.faa.gov/uas/, USA, (31 March 2016).

Gartner Group, 2015. Smartphone sales press release (http://www.gartner.com/newsroom/id/3169417), November 2015.

Guha-Sapir D., Hoyois, Ph., Below R., 2013. "Annual Disaster Statistical Review 2012: The number and trends", Brussels: CRED.

Hamp, Q., Zhang, R., Chen, L., Gorgis, O., Ostertag, T., Loschonsky, M., Reindl, L., 2014. "New technologies for the search of trapped victims", Ad Hoc Networks, Vol. 13, pp 6982.

Lefeuvre, F., Tanzi, T. J., 2013. "International Union of Radio Science, International Council for Science (ICSU), Joint Board of Geospatial Information Societies (jBGIS)," in United Nations office for outer Space Affairs (OOSA).

Malone, B. L., et al., 2006. "Hurricane Katrina Final Report", Wireless Emergency Response Team (WERT).

Meier, P., 2014. Humanitarians in the Sky: Using UAVs for Disaster Response, http://irevolution.net/2014/06/25/ June 25, 2014, last viewed, 30 June 2014.

Moussa, M., Youssef, M., 2009. "Smart Devices for Smart Environments: Device-free Passive Detection in Real Environments", IEEE International Conference on Pervasive Computing and Communications, Galveston, TX, pp.1-6.

Pedersen, J., 2014. Use of UAVs in the NGO World, CRS Conference - ICT4Development, Nairobi, Kenya, March 25-28.
Polat, B., K., Sachdeva, P., Ammar, M. H., Zegura, E., W., 2011. "Message ferries as generalized dominating sets in intermittently connected mobile networks", Elsevier Journal on Pervasive and Mobile Computing,7(2): 189-205.

Sébastien, O., Harivelo, F., 2015. "Enhancing Disaster Management by Taking Advantage of General Public Mobile Devices: Trends and Possible Scenarios", in "Wireless Public Safety Networks volume 1: Overview and Challenges." D. Camara \& N. Nikaein edt., Chapter 9, 261-296. ISTE Press Elsevier London (UK).

Stratxx, 2014. News Release, http://www.stratxx.com/blog/archive/ 2014/03/19/, (30 June, 2014).

Tanzi, T.J., Perrot, P., 2009. Télécoms pour l'ingénierie du risque (in French), éditions hermès ed. Collection Technique et Scientifique des Télécoms, Paris, France.

Tanzi, T. J., Lefeuvre, F., 2010. "Radio sciences and disaster management", C.R. Physique 11, 114-224.

Tanzi, T. J., Lefeuvre, F., 2011. "The Contribution of Radio Sciences to Disaster Management," in International Symposium on Geo-information for disaster management (Gi4DM 2011), Antalya, Turkey.

Tanzi, T. J., Isnard, J., 2014. "Robot d'intervention multifonction d'assistance post-catastrophe. Réflexions sur un drone " humanitaire "," Revue de l'Electricité et de l'Electronique (REE), pp. 24-30.

Wang, W., Joshi, R., Kulkarni, A., Leong, W.K., Leong, B., 2013. "Feasibility Study of Mobile Phone WiFi Detection in Aerial Search and Rescue Operations", APSys.

Wilkinson, P., Cole, D., 2010. "The Role of the Radio Sciences in the Disaster Management," Radio Science Bulletin, vol. 3358 , pp. 45-51.

Zorn, S., Maser, M., Goetz, A., Rose, R., Weigel, R., 2011. "A power saving jamming system for E-GSM900 and DCS1800 cellular phone networks for search and rescue applications", WiSNet. 